\title{
How Different Reading Habits Influence Lines, Words and Pseudowords Bisection: Evidence from Italian and Hebrew
}

\author{
Chiara Valeria Marinelli1 ${ }^{*}$, Lisa S. Arduino ${ }^{2,3}$, Inbar L. Trinczer ${ }^{4}$, Naama Friedmann ${ }^{4}$ \\ ${ }^{1}$ Department of History, Society and Human Studies, University of Salento, Lecce, Italia \\ ${ }^{2}$ Department of Human Sciences, Lumsa University, Roma, Italia \\ ${ }^{3}$ Institute of Cognitive Sciences and Technologies-CNR, Roma, Italia \\ ${ }^{4}$ Language and Brain Lab School of Education, Tel Aviv University, Tel Aviv, Israel \\ Email: *chiaravaleria.marinelli@unisalento.it
}

How to cite this paper: Marinelli, C. V., Arduino, L. S., Trinczer, I. L., \& Friedmann, N. (2019). How Different Reading Habits Influence Lines, Words and Pseudowords Bisection: Evidence from Italian and Hebrew. Psychology, 10, 2051-2061. https://doi.org/10.4236/psych.2019.1015132

Received: March 29, 2019

Accepted: December 20, 2019

Published: December 23, 2019

Copyright (c) 2019 by author(s) and Scientific Research Publishing Inc. This work is licensed under the Creative Commons Attribution International License (CC BY 4.0).

http://creativecommons.org/licenses/by/4.0/

\begin{abstract}
When young adult readers bisect a visually presented line, they generally posit the subjective midpoint to the left of the objective one, a phenomenon called pseudoneglect. This phenomenon also pertains to orthographic material (words, pseudowords, consonant strings) and recently it has been shown that stimulus length may affect the bisection of lines and orthographic material differently: whereas lines are always bisected leftward, orthographic material is sensitive to length showing an opposite rightward bisection bias only with short stimuli. Pseudoneglect is generally ascribed to two main factors: cerebral asymmetries in visuo-spatial tasks and reading habits. To investigate this issue, 46 adults with different directional reading habits (Italian and Hebrew-speaking) were asked to bisect lines, words, and pseudowords of different lengths in both languages. Stimuli were presented on the screen of a tablet computer and the participants marked the target midpoint with their forefinger. The results showed that reading habits have an important role in the magnitude and direction of the bias in bisecting verbal and nonverbal material.
\end{abstract}

\section{Keywords}

Line and Word Bisection, Reading Habits, Hebrew-Speaking Vs. Italian Speaking

\section{Introduction}

When left-to-right readers are required to bisect a line, they posit the subjective 
midpoint to the left of the true objective one (e.g., Bradshaw et al., 1986) This phenomenon is called pseudoneglect (Bowers \& Heilman, 1980), because of its similarity to the deviation from the middle shown by unilateral neglect patients in line bisection (e.g., Heilman et al., 1993), although in the opposite direction.

At least two different mechanisms have been considered to be responsible for pseudoneglect: the first is the differential involvement of the cerebral hemispheres when performing a bisection task (e.g., Gallace et al., 2008). Indeed, studies on patients with unilateral spatial neglect (Heilman et al., 1993) and neuroimaging studies with unimpaired participants (e.g., Cicek et al., 2009) testified the contribution of the right intra-parietal sulcus and the lateral peristriata cortex when computing a line bisection task. The magnitude of the leftward bias has been shown to be related to the rightward hemispheric lateralization (Zago et al., 2017), as well as to individual laterality factors (such as handedness and eye sighting dominance) (Ochando \& Zago, 2018). The second mechanism relates to the reading direction (e.g., Chokron et al., 1993; Gabay et al., 2013; Girelli et al., 2017; Rinaldi et al., 2014). In a study among normal right-handed adults differing by their reading habits, French and Hebrew-speaking participants were characterized by opposite patterns of deviations in line bisection (Chokron \& Imbert, 1993): while French participants showed a leftward bias, Hebrew-speaking ones made a significant rightward deviation error. Such finding was further corroborated by studies in which pre-school children, belonging to languages with opposite reading habits, were examined (Chokron \& De Agostini, 1995; Fagard \& Dahmen, 2003).

Pseudoneglect even characterizes the bisection of visual strings such as words and pseudowords (Fischer, 1996, 2000a, 2000b) and it has been shown to be partly independent from variables such as the kind of task (visual or motor bisection tasks, Arduino et al., 2012), the request to read aloud or not the stimuli (Fischer, 2000b), the type of language (Fischer, 2000b), the different grammatical word classes (Fischer, 2000b) or the font type and size used (Fischer, 2004). Arduino et al. (2010) conducted a study on unimpaired young adult Italian readers showing that orthographic strings and lines behaved differently with respect to stimulus length: whereas short and long lines were always bisected with a similar leftward bias, orthographic strings (like words and pseudowords) yielded a similar leftward bias when longer, but were all bisected towards the right of the objective center when relatively short. With respect to word bisection, both the imbalanced hemispheric activation hypothesis (e.g., Scarisbrick et al., 1987) and the reading habits hypothesis may account for the reported leftward bias, by postulating that similar mechanisms are involved in the bisection of both lines and words. However, such theories fail to account for the rightward bias in shorter Italian words. An alternative view is offered by the Attentional Scaling Hypothesis by Fischer (1996) who postulates that the bisection of orthographic material obeys to an additional lexical mechanism, such as lexical access, which is independent and somehow additive to the mechanisms involved in line bisection. Following this line, the bisection difference between short and long words 
could be explained by the optimal viewing position which is located in different parts of the strings, depending in their length. However, it is worth noting that in Arduino et al. (2010) nonverbal symbol strings yielded a similar result to letter strings, despite the lack of verbal information and thus the unnecessary lexical entry, and the suggestion was made that this effect is partially due to the high perceptual similarity of orthographic letter strings and nonverbal symbol strings, both constituted by discrete elements when compared to continuous solid lines. For this reason, the argument was made that the difference between lines and strings emerges early on in a low-level pre-processing step that detects the presence or absence of space between characters (words, pseudowords, strings of hashes, and dotted lines all shared this property, unlike solid lines) and that only later the verbal nature of the stimulus emerges (Girelli et al., 2018). Indeed, words bisection is also driven by lexical variables as showed by the pioneering studies by Fisher (e.g., 2000) and more recently by Veronelli et al. (2014). The authors found a reduced rightward bias in bisecting irregularly stressed short words (i.e., with stress on the antepenultimate syllable instead on the penultimate syllable) indicating that word stress location may attract an orient visuo-spatial attention.

In the present study, we compared two populations differing in their reading habits: Italian (from left-to-right) and Hebrew-speaking participants (from right-to-left). Participants were required to bisect lines and orthographic stimuli in both languages: Italian participants bisected both Italian and Hebrew words and pseudowords and vice versa. If reading habits have a role in modulating the bias direction (leftward or rightward), predictions could be made that $\mathrm{He}$ brew-speaking participants were expected to bisect words, and possibly also lines, more rightward compared to Italian participants. If, however, both reading habits and cerebral hemispheric specialization contribute to the bisection bias, we would expect the two factors to work in opposite directions in $\mathrm{He}$ brew-speaking participants, yielding a bisection bias more located to the right than in Italian, but not necessarily to the right of the middle. Regarding the bisection of stimuli not in their mother tongue, we expected a large rightward bias for Italian subjects in bisecting Hebrew stimuli (due to the fact that for Italians, Hebrew words are similar to symbols). On the contrary, for Hebrew-speaking participants, a smaller rightward bias with Italian characters was expected, since even if Italian words are not known, they are still recognized as strings made up of orthographic elements. Indeed, most Hebrew-speakers read and speak at least one left-to-right language correctly (e.g., English, French) and this may attenuate the right-to-left bias connected to the reading habits.

\section{Method}

\subsection{Participants}

Twenty-eight Italian-speaking and $28 \mathrm{Hebrew}$-speaking adults participated in the study (see Table 1). Participants were $1^{\text {st }}-3^{\text {rd }}$ years students at the LUMSA 
Table 1. Socio-demographic characteristics of Italian and Hebrew participants.

\begin{tabular}{ccccc}
\hline & Italian participants & Hebrew participants & difference & $\mathrm{p}$ \\
\hline Male & 8 & 12 & $\chi^{2}=1.24$ & 0.26 \\
Female & 20 & 16 & & \\
$\begin{array}{c}\text { Age: } \text { mean years } \\
\text { (standard deviation) }\end{array}$ & $23.2( \pm 3.6)$ & $20.3( \pm 3.7)$ & $\mathrm{t}(55)=1.69$ & 0.10 \\
\hline
\end{tabular}

University of Rome and the University of Tel Aviv. As reported in Table 1, groups were comparable for age and gender. All participants had no history of reading or spelling disorders and normal or correct vision. All participants were right-handed.

Prior to start the experiment, all of them provided an informed consent and the study was approved by the Ethic committee of both the Universities.

\subsection{Procedure}

The research was conducted using a tablet computer (Samsung Galaxy Tab P7510, 10.1 inches, screen resolution 800X200; Android OS v. 3.2 operating system), for which an application ("Emtzà Emtza v1.0") has been specifically designated for stimuli presentation and data collection.

The stimuli appeared on the screen, one at a time, and the participants were asked to bisect each of them with their right hand index finger from top to bottom, as quickly and accurately as possible. Participants were asked to find the centre of the stimuli, with the precise instruction to look at the stimulus as a visual object and not counting the number of letters in the case of orthographic materials. The application presented a single stimulus at a time, in the middle of the screen. The experiment began with 10 practice stimuli, which were used to exemplify the bisection request, and to allow for adapting to the task. The practice stimuli consisted of object pictures to support the request for finding the visual center of various objects.

The experimental stimuli appeared on the screen for an unlimited time, and disappeared only after the participant's response. A $500 \mathrm{~ms}$ delay between stimulus bisection completion and the appearance of the next stimulus was used. The stimuli appeared in black on a white screen. The tablet was placed on a desk in front of the subjects, approximately $40 \mathrm{~cm}$ away from their eyes. Words and pseudowords were presented in lowercase Courier New font, equivalent to 53 points; the width of line-type stimuli is $1 \mathrm{~mm}$.

The participants did not receive any response-contingent feedback from the experimenter during tests. However, throughout the examination, if the participant did not bisect the stimulus as instructed (from top to bottom), or crossed the boundaries throughout the bisection, the mark was not recorded, leaving the stimulus presented on the screen until the subject correctly bisected the stimulus.

At the end of the tests' administration, the system provided a separate output 
for each participant. The employment of the tablet facilitated a more reliable measurement than any manual means. Several indices were collected for each stimulus: its serial number according to the various tests, its appearance in the random order for that subject, the stimuli's length, its true center, and the point where the subject bisected it, namely, the subject mark's degree of deviation from the stimulus' true center. Computation of participants' marking deviation was administered by subtracting stimulus' real centre from the mark's placement, such that the right deviation from the bisection line was defined by positive values, while a left deviation with a negative values, similarly to the number axis. All stimuli were measured in pixels. For clarity and simplicity in this article results are presented in $\mathrm{mm}$ (pixel data were converted in millimetres by multiplying by 0.17 ). Outliers (i.e., data above/below 4 standard deviations of the subject average for each stimulus type) were eliminated.

Each participant was examined separately, in a quiet room, under best lighting conditions. The participants were not previously provided with information concerning the research's purpose.

\subsection{Stimuli}

Stimuli were lines, Hebrew words and pseudowords and Italian words and pseudowords. Both Italian and Hebrew-speaking participants performed the task both in their native language and in the other, unknown, language. All stimuli were randomized, and every 1 - 3 stimuli a filler appeared.

Lines: 32 horizontal lines of two different lengths (corresponding to the length - in pixel-to the orthographic stimuli were used (one to one to the words and pseudowords stimuli). The length of the lines was $40.9 \pm 1.5 \mathrm{~mm}$ for short lines and $86.8 \pm 3.1 \mathrm{~mm}$ for long lines.

Pseudowords. Sixteen pronounceable and orthographically legal nonwords or pseudowords, 8 short (4-letters) and 8 long (8-letters) were used for both languages and were matched for length. In Hebrew, for each length, we used four pseudowords that retain the Hebrew morphological structure and four that were legal letter strings.

Words: 16 Italian words and 16 Hebrew words were used: 8 were short $(3$ - 5 letters, with a mean of 4 letters) and 8 were long ( 7 - 9 letters, with a mean of 8 letters words). In Italian, words were all morphologically simple nouns. In Hebrew, 3 letter words included only the 3-consonantal root, and 5, 7, and 9 letter words included the root in the center with a prefixes before the root and a suffix after the root (e.g., the 7 letter word תתפרקנה, TTPRKNH, included the prefix " $t \mathrm{t}$ ", the root prk, and the suffix "nh"). Because we wanted to only include words in which the root appears in the middle, so as to avoid the effect of the root's position on bisection (see Trinczer \& Friedmann, 2014), we only used Hebrew words in which the root was in the exact center, with the same number of letters in the prefix and suffix.

Fillers. 52 fillers were used in order to prevent fixation of the participant's hand in the tablet's center. The fillers were similar to stimuli in the different tests 
but, unlike the test stimuli, appeared in various random positions on the screen. The information for filler bisection was not analyzed.

\subsection{Data Analysis}

We analysed separately the effects on line and orthographic bisection. In both analyses, the dependent variable was the deviation from the stimulus' centre for each participant and each target. The first analysis is a repeated measures ANOVA with length (short vs long) as repeated measure and language group (Italian vs Hebrew-speaking participants) as between variable. The second analysis is a repeated measures ANOVA with length (short vs long), lexicality (words vs pseudowords), and character (Italian vs Hebrew) as repeated measure and language group (Italian vs Hebrew-speaking participants) as between variable. Significant interactions were explored by Bonferroni post hoc test.

\section{Results}

\section{Line bisection}

Line bisection showed a significant main effect of language group $[\mathrm{F}(1,54)=$ 6.59, $p=0.013$ ] indicating a rightward bias for Hebrew-speaking participants $(+0.30 \mathrm{~mm}$ to the right of the centre) and a leftward bias for Italian participants $(-0.26 \mathrm{~mm}$, namely, $0.26 \mathrm{~mm}$ to the left of the centre).

Language interacted with length $[\mathrm{F}(1,54)=9.35, p=0.003]$ as the bisection bias for short lines was similar for Italian and Hebrew-speaking participants, whereas in long lines a different bias emerged $(p=0.088)$. Hebrew-speaking participants showed a rightward bias $(+0.67 \mathrm{~mm})$ significantly different from zero $\left(\mathrm{t}_{(55)}=2.85, p=0.006\right)$ in the case of long lines and a leftward bias $(-0.06 \mathrm{~mm})$ not significantly different from zero $(\mathrm{t}<1)$ for short lines. Italian participants showed a leftward bias that did not differ significantly from zero for both short $(-0.16 \mathrm{~mm})$ and long $(-0.36 \mathrm{~mm})$ lines. See Figure 1.

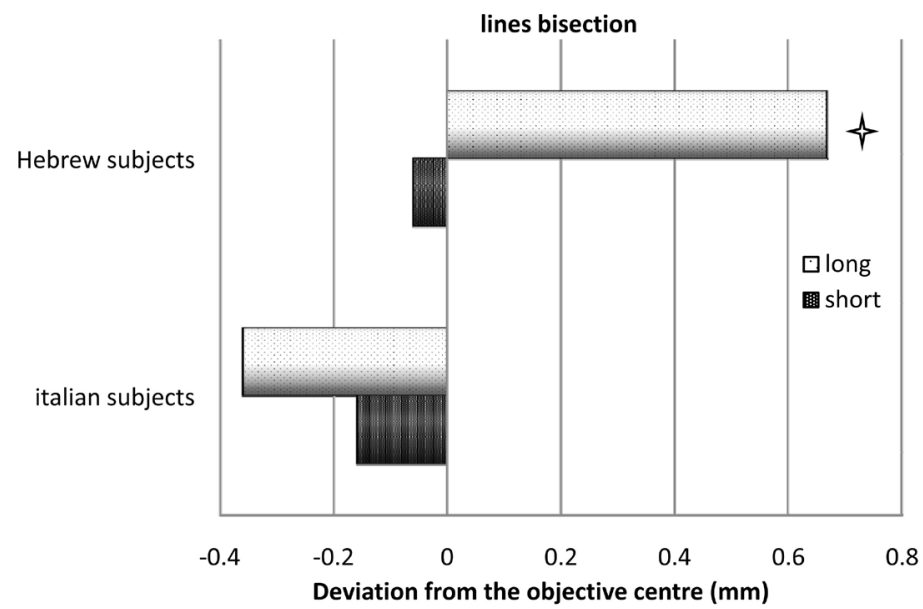

Figure 1. Line Bisection. Deviation for the objective centre (in $\mathrm{mm}$ ) of Hebrew-speaking and Italian participants in bisecting short and long lines. The asterisks identify a significant deviation from zero according to $t$ test. 
Results regarding line bisection were in accordance to the literature: in Hebrew-speaking participants, a rightward bias was evident while in Italians the opposite leftward bias was obtained. Such cross-linguistic differences were particularly evident in the bisection of long lines.

Bisection of orthographic materials

Figure 2 illustrates the results of the orthographic bisection task. There was a significant main effect of length $[\mathrm{F}(1,54)=11.19, p=0.001)]$ and of character $[\mathrm{F}$ $(1,54)=82.20, p<0.0001]$. The main effect of language group was not significant.

Length interacted with character $[\mathrm{F}(1,54)=9.56, p=0.003]$ and lexicality $[\mathrm{F}$ $(1,54)=4.69, p=0.035]$. The length $\mathrm{x}$ character interaction showed a larger rightward bias for longer stimuli only for Hebrew characters $(+1.02 \mathrm{~mm}$ difference between the biases measure in short and long Hebrew stimuli) but not for Italian characters $(0.30 \mathrm{~mm}, p<0.0001)$. Such larger rightward bias for Hebrew relative to Italian characters was even evident for the short stimuli $(+0.51 \mathrm{~mm}$ vs

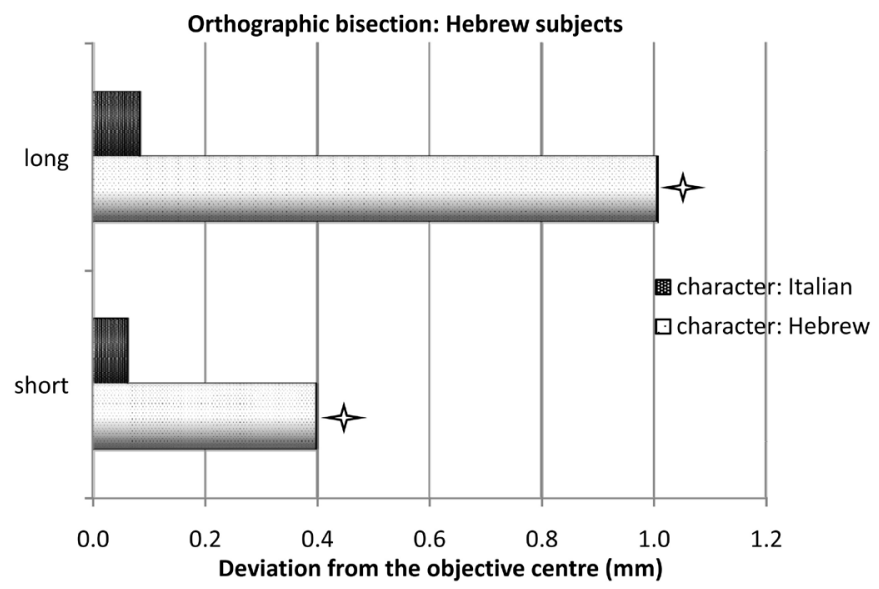

(a)

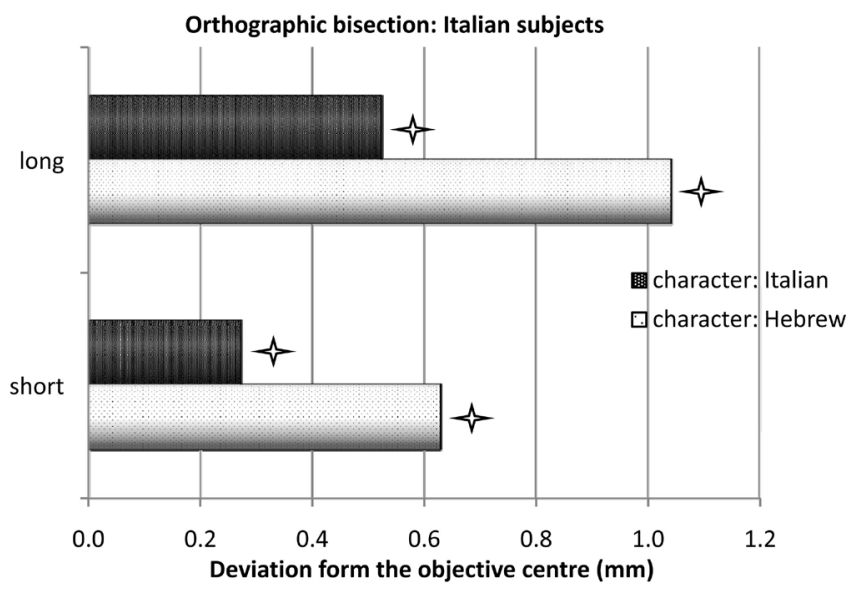

(b)

Figure 2. Orthographic stimuli bisection. Deviation from the objective centre (in $\mathrm{mm}$ ) of Hebrew-speaking (a) and Italian participants (b) in bisecting short and long orthographic stimuli. The asterisks identify a significant deviation from zero according to $t$ test. 
$0.17 \mathrm{~mm}$ respectively, $p=0.001)$. The length $\mathrm{x}$ lexicality interaction showed lexicality effect for long ( $p=0.049)$ but not for short stimuli; length effect was significant both for words (mean of difference between long and short $=0.22 \mathrm{~mm}$; $p=0.012$ ) and for pseudowords (mean difference $=0.43 \mathrm{~mm}, p<0.0001$ ), but it was larger in pseudowords.

Length $\times$ character $\times$ language interaction approached significance $[\mathrm{F}(1,54)=$ 3.08, $p=0.057$ ]. Figure 2(a) illustrates that Hebrew-speaking participants showed a significant rightward bias for orthographic stimuli of both lengths when written in their own language (short $+0.40 \mathrm{~mm}$; $\mathrm{t}(55)=3.89, p<0.0001$; long $+1.01 \mathrm{~mm}, \mathrm{t}(55)=5.21, p<0.0001)$. By contrast, when Hebrew-speaking participants were required to bisect Italian words and pseudowords, the bias was close to zero for both short $(+0.06 \mathrm{~mm}$; $\mathrm{t}$ about 1$)$ and long $(+0.08 \mathrm{~mm} ; \mathrm{t}<1)$ stimuli.

The Italian participants (see Figure 2(b)) showed a significant and large rightward bias for stimuli written in Hebrew, both for short $(+0.63 \mathrm{~mm} ; \mathrm{t}(55)=$ $4.15, p<0.0001)$ and long stimuli $(+1.04 \mathrm{~mm}$; $\mathrm{t}(55)=4.96, p<0.0001)$; a significant rightward bias was also evident when Italians bisected Italian characters for both short $(+0.27 \mathrm{~mm} ; \mathrm{t}(55)=1.91, p=0.052)$ and long stimuli $(+0.52 \mathrm{~mm}$; $\mathrm{t}(55)=2.60, p=0.012)$.

A post hoc exploration of the interaction showed a length effect for Hebrew-speaking participants in bisecting Hebrew stimuli (difference in the rightward bias between long and short stimuli $=0.61 \mathrm{~mm} ; p<0.0001$ ), whereas the effect of length was not significant for Hebrew-speaking participants when reading Italian stimuli (difference $=0.02 \mathrm{~mm}$ ); Italian-speaking participants showed a significant length effect for Hebrew stimuli (difference $=0.41 \mathrm{~mm}, p=$ $0.034)$, and a non-significant tendency when bisecting characters of their own language (difference $=0.25 \mathrm{~mm} ; p>0.1$ ). Hebrew stimuli generally produced a larger rightward bias than did the Italian stimuli. This difference was significant only for the long stimuli, both for the Hebrew-speaking participants $(0.92 \mathrm{~mm} ; p$ $<0.0001)$ and for Italians $(0.52 \mathrm{~mm} ; p=0.002)$.

Cross-linguistic differences also emerged when participants were required to bisect orthographic material. Overall, Hebrew-speaking participants showed a rightward bias when bisecting orthographic stimuli of their own language (for both short and long words and pseudowords). On the contrary, Italian words and pseudowords were bisected by Hebrew-speaking participants more closely to the middle, irrespectively of the stimulus' length.

By contrast, Italian participants showed a rightward bias when bisecting Italian orthographic stimuli as well as stimuli presented in Hebrew characters. Length seemed to affect bisection more for Hebrew character than for Italian ones.

\section{Conclusion}

In the present study Italian and Hebrew-speaking adults bisected lines and orthographic stimuli in the two languages. The results with lines replicated well 
known findings (e.g., Arduino et al., 2010; Chokron \& Imbert, 1993), where Italians showed a leftward bisection bias and Hebrew-speaking participants a rightward one. This finding reassured us about the validity of the tablet procedure. The finding that Hebrew-speaking participants bisected short lines more correctly (i.e., closer to the real centre of the line) could provide some evidence of an attenuation of the bias due to the interaction between cerebral asymmetries and reading habits mechanisms. Indeed, and differently from Italians, in right-to-left readers the two mechanisms are in opposition: the reading habits bring spatial attention to right side of the string whereas the hemispheric specialization takes to the left. This conflict could be solved with a compromise and resulting in a more accurate bisection (see Fagard \& Dahmen, 2003, for a similar conclusion).

Data regarding word and pseudoword bisection were more puzzling, especially for the Italian data. Importantly, Hebrew-speaking participants confirmed our predictions in bisecting orthographic material, by positing the subjective midpoint significantly to the right when bisecting orthographic strings in their mother language. When the same Hebrew-speaking participants were requested to bisect Italian words and pseudowords, they showed a leftward bias, further confirming the role of reading habits in such a task. In fact, even if Hebrew-speaking participants do not speak Italian (for them Italian words have not lexical status), they know English and are hence acquainted with left-to-right reading with these characters. These results showed that the cerebral dominance of the right hemisphere cannot account for the findings by itself, because it is more a combination between the hemisphere dominance and reading habits. When orthographic stimuli are presented (independently from the lexical status), they are processed and treated at least according to the language in which they are presented. The present finding confirms the pre-lexical nature of the early processes affecting attentional bias (Girelli et al., 2018).

The Italian data regarding the bisection of words were quite different from previous results, where only Italian language was tested (see e.g., Arduino et al., 2010; as well as healthy subjects examined in Arduino et al., 2012 and Veronelli et al., 2014). We did not replicate the leftward bias for words and pseudowords, in that a strong rightward bias was present in the Italian participants' performance when bisecting both Italian and Hebrew letter strings. The results on $\mathrm{He}$ brew character are not surprising, in that in a recent study by Girelli et al. (2018) it has been shown that Italian participants bisected non-orthographic strings more rightward. We can assume that Hebrew characters are not recognized as letters by the Italians and that they were treated as non-orthographic strings. The fact that a rightward bias was present even in bisecting Italian orthographic strings could be due to the fact that in this study all stimuli were mixed together and, as shown by Arduino et al. (2010), the pseudoneglect is less marked, if not absent, in this condition.

In conclusion, we confirm our prediction regarding a reading habits role in bisecting lines, graphic and orthographic strings, and the fact that such vi- 
suo-spatial materials underline partially different mechanisms.

\section{Conflicts of Interest}

The authors declare no conflicts of interest regarding the publication of this paper.

\section{References}

Arduino, L. S., Marinelli, C. V., Pasotti, F., Ferrè, E., \& Bottini, G. (2012). Representational Neglect for Words as Revealed by Bisection Tasks. Journal of Neuropsychology, 6, 43-64. https://doi.org/10.1111/j.1748-6653.2011.02003.x

Arduino, L. S., Previtali, P., \& Girelli, L. (2010). The Centre Is Not in the Middle: Evidence from Line and Word Bisection. Neuropsychologia, 48, 2140-2146. https://doi.org/10.1016/j.neuropsychologia.2010.04.005

Bowers, D., \& Heilman, K. M. (1980). Pseudoneglect: Effects of Hemispace on a Tactile Line Bisection Task. Neuropsychologia, 18, 491-498. https://doi.org/10.1016/0028-3932(80)90151-7

Bradshaw, J. L., Bradshaw, J. A., Nathan, G., Nettleton, N. C., \& Wilson, L. E. (1986). Leftwards Error in Bisecting the Gap between Two Points: Stimulus Quality and Hand Effects. Neuropsychologia, 24, 849-855. https://doi.org/10.1016/0028-3932(86)90084-9

Chokron, S., \& De Agostini, M. (1995). Reading Habits and Line Bisection: A Developmental Approach. Cognitive Brain Research, 3, 51-58. https://doi.org/10.1016/0926-6410(95)00018-6

Chokron, S., \& Imbert, M. (1993). Egocentric Reference and Asymmetric Perception of Space. Neuropsychologia, 31, 267-275. https://doi.org/10.1016/0028-3932(93)90091-D

Chokron, S., Perenin, M. T., Rode, G., \& Imbert, M. (1993). Scanning Direction and Line Bisection. Perception, 22, 120.

Cicek, M., Deouell, L., \& Knight, R. T. (2009). Brain Activity during Landmark and Line Bisection Tasks. Frontiers in Human Neuroscience, $3,7$. https://doi.org/10.3389/neuro.09.007.2009

Fagard, J., \& Dahmen, R. (2003). The Effects of Reading-Writing Direction on the Asymmetry of Space Perception and Directional Tendencies: A Comparison between French and Tunisian Children. Laterality, 8, 39-52. https://doi.org/10.1080/713754473

Fischer, M. H. (1996). Bisection Performance Indicates Spatial Word Representations. Cognitive Brain Research, 4, 163-170. https://doi.org/10.1016/S0926-6410(96)00029-8

Fischer, M. H. (2000a). Word Centre Is Misperceived. Perception, 29, 337-354.

Fischer, M. H. (2000b). Perceiving Spatial Attributes of Print. In A. Kennedy, R. Radach, D. Deller, \& J. Pynte (Eds.), Reading as a Perceptual Process (pp. 89-117). Amsterdam: Elsevier. https://doi.org/10.1016/B978-008043642-5/50007-3

Fischer, M. H. (2004). Orthographic Contributions to Perceived Word Center. Brain and Language, 88, 321-330. https://doi.org/10.1016/S0093-934X(03)00163-9

Gabay, Y., Gabay, S., Schiff, R., Ashkenazi, S., \& Henik, A. (2013). Visuospatial Attention Deficits in Developmental Dyslexia: Evidence from Visual and Mental Number Line Bisection Tasks. Archives of Clinical Neuropsychology, 28, 829-836.

https://doi.org/10.1093/arclin/act076

Gallace, A., Imbornone, E., \& Vallar, G. (2008). When the Whole Is More than the Sum of the Parts: Evidence from Visuospatial Neglect. Journal of Neuropsychology, 2, 387-413. https://doi.org/10.1348/174866407X252639 
Girelli, L., Marinelli, C. V., Grossi, G., \& Arduino, L. S. (2017). Cultural and Biological Factors Modulate Spatial Biases over Development. Laterality, 22, 725-739. https://doi.org/10.1080/1357650X.2017.1279623

Girelli, L., Previtali, P., \& Arduino, L. S. (2018). What Makes a Word So Attractive? Disclosing the Urge to Read While Bisecting. British Journal of Psychology, 109, 862-878. https://doi.org/10.1111/bjop.12303

Heilman, K. M., Watson, R. T., \& Valenstein, E. (1993). Neglect and Related Disorders. In K. M. Heilman, \& E. Valenstein (Eds.), Clinical Neuropsychology (3rd ed., pp. 279-336). New York: Oxford University Press.

Ochando, A., \& Zago, L. (2018). What Are the Contributions of Handedness, Sighting Dominance, Hand Used to Bisect, and Visuospatial Line Processing to the Behavioral Line Bisection Bias? Frontiers in Psychology, 9, 1688. https://doi.org/10.3389/fpsyg.2018.01688

Rinaldi, L., Di Luca, S., Henik, A., \& Girelli, L. (2014). Reading Direction Shifts Visuospatial Attention: An Interactive Account of Attentional Biases. Acta Psychologica, 151, 98-105. https://doi.org/10.1016/j.actpsy.2014.05.018

Scarisbrick, D. J., Tweedy, J. R., \& Kuslansky, G. (1987). Hand Preference and Performance Effects on Line Bisection. Neuropsychologia, 25, 695-699.

https://doi.org/10.1016/0028-3932(87)90061-3

Trinczer, I. L., \& Friedmann, N. (2014). Who Moved the Middle? Effect of Reading Direction and Morphology on Visual Perception of Written Words. Presented at the International Neuropsychological Society Meeting, Jerusalem.

Veronelli, L., Vallar, G., Marinelli, C. V., Primativo, S., \& Arduino, L. S. (2014). Line and Word Bisection in Patients with Unilateral Spatial Neglect. Experimental Brain Research, 232, 133-146. https://doi.org/10.1007/s00221-013-3726-7

Zago, L., Petit, L., Jobard, G., Hay, J., Mazoyer, B., Tzourio-Mazoyer, N. et al. (2017). Pseudoneglect in Line Bisection Judgment Is Associated with a Modulation of Right Hemispheric Spatial Attention Dominance in Right-Handers. Neuropsychologia, 94, 75-83. https://doi.org/10.1016/j.neuropsychologia.2016.11.024 